\title{
Structure Optimization Based on 3d Modeling to a New Hydraulic Boarding Ladder
}

\author{
Jing Liu, Meiping $\mathrm{Wu}$, Jun Zhang \\ School of Mechanical Engineering \\ Jiangnan University
}

\begin{abstract}
The existing boarding ladders for maritime operations, loading and unloading of passengers are with the problems of complicated structures, low level of automation and even safety problems. Aiming at solving the problems, this paper reseraches and develops a new type of hydraulic boarding ladder and models it by CATIA. The working principle and the luffing $\&$ rotary structure are introduced as well.
\end{abstract}

Keywords-Hydraulic boarding ladder, Working principle, Modeling, Structure Optimization

\section{INTRODUCTION}

At the present time, most wharfs use simple boarding ladders for maritime operations, loading and unloading of passengers[1]. But the existing boarding ladder is not so good because of its complicated structure, low level of automation, and poor reliability[2][3]. Furthermore, it is not safe for the elder passengers to use the simple boarding ladder.

This paper develops a Boarding ladder with luffing \& rotary structure. It not only gets rid of the shortages of the simple boarding ladder in China but also has many improvement and optimization. Such as reliable and stable structure, high level of automation, advanced security capabilities and so on.

\section{THE STRUCTURE AND WORKING PRINCIPLE OF THE NEW BOARDING LADDER}

With the development of science and Technology, some new types of hydraulic boarding ladders have been applied to maritime operations, loading and unloading passengers etc. The structure of a simple boarding ladder is showed in the following figure [4][5]:

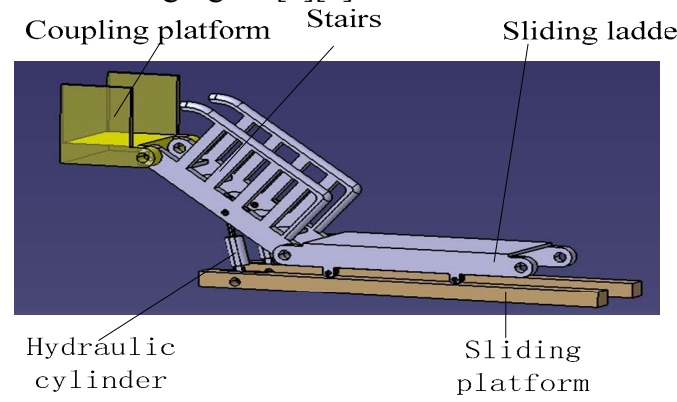

Figure1. Existing structure of boarding ladder

In figure1, the structure of the boarding ladder has been improved enormously. But the structure is not compact or stable. In addition, affected by the wind and waves the ship may bob up and down, move from side to side or complex both motions. This obviously cannot coincide with the ships' movement in all weather conditions. So the boarding ladder is not seaworthy applying. All these shortages limit its application scope.

Based on the analysis of the mentioned boarding ladder above, this paper presents a new hydraulic boarding ladder. The structure is showed in the following figure:

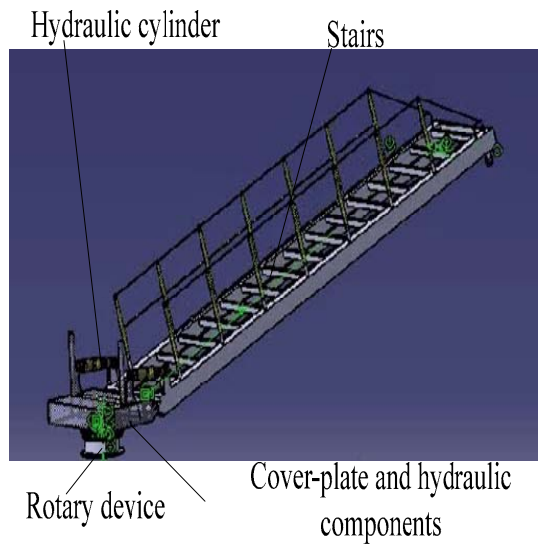

Figure 2. The whole structure of new boarding ladder

The length of the ladder is $9.6 \pm 0.5 \mathrm{~m}$, the width is about $1 \mathrm{~m}$. A pair of castor wheels is fixed on the fore-end of the ladder, by which the ladder can slide on the pier.

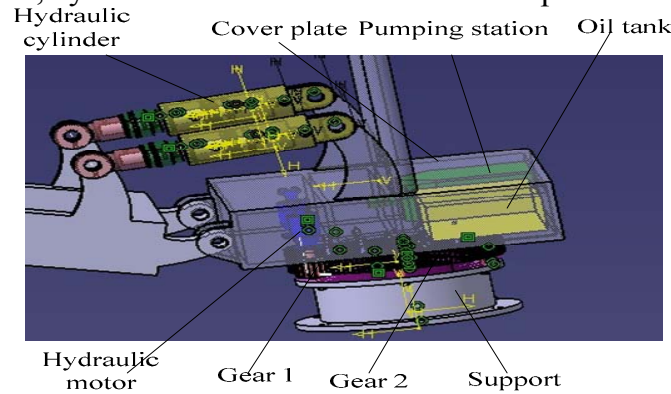

Figure 3. Luffing structure and rotary Structure

Figure. 2 and Figure. 3 show that the new hydraulic boarding ladder is controlled by a pair of hydraulic cylinders[6] to execute the ladder's up and down and a pair of hydraulic motors is used to control the ladder's rotation.

Compared with the structure showed in Figure.1, the structure showed in Figure. 2 and Figure. 3 is more reliable while the boarding ladder is more practical. 
Because of the great mass of the new ladder, the paper chooses swiveling gear to bear great overturning moment.

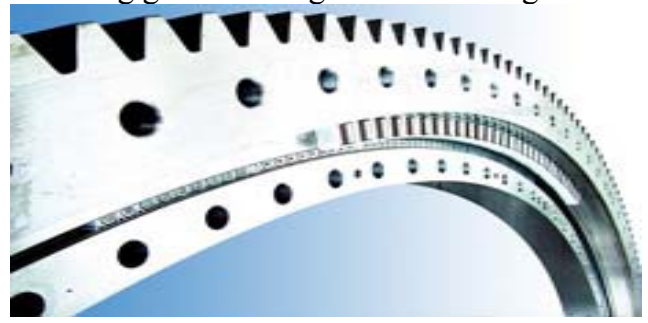

Figure 4. Swiveling gear

The swiveling gear is fixed on the pedestal with flange. The installation is showed in the following figure.

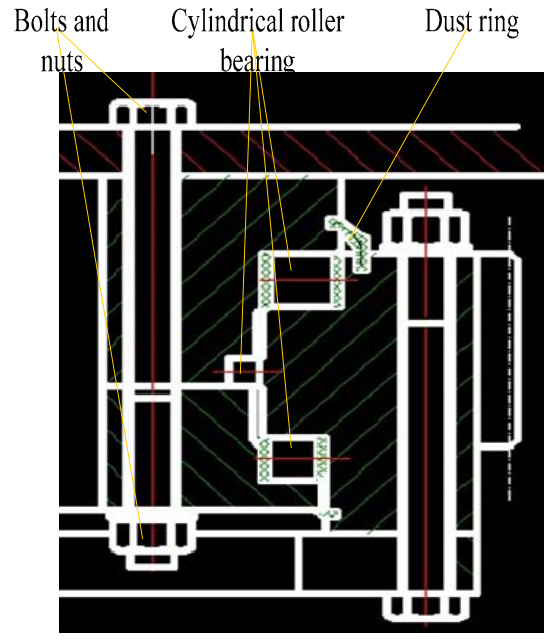

Figure 5. Installation of swiveling gear

\section{ANALYSIS AND DESIGN OF THE HYDRAULIC SYSTEM}

\section{A. the function of the hydraulic system}

Because of the complicated situations at sea, the ship may rocked from side to side, bob up and down on water as waves pass by. The hydraulic system of the boarding ladder must realize two functions, one is moving from side to side, and the other bobbing up and down and also the combination both types. In a word, the hydraulic system should realize the following movements, which are:

1) Can tilt to be a slope, and the angle range of the slope is $\pm 30^{\circ}$.

2) Can rotate, and the range of the rotating angle is about $\pm 180^{\circ}$.

3) Make the ladder bob on the water up and down as waves pass by.

4) Can wobble in case that it cracked to pier or some heavy objects else. This may cause the pressure of hydraulic system to be very high, and even cause some damage to the ladder.

5) When the ladder tilts, it can stop at any angle in the range of $\pm 30^{\circ}$

6) When the ladder rotates, it can stop at any angle in the range of $\pm 180^{\circ}$.
7) Should control the ladder to move smoothly.

$B$. main parameters of the hydraulic system

Working pressure: $12 \mathrm{MPa}$

Hydraulic motor: NHM2-200.

Discharge: $0.2 \mathrm{~L} / \mathrm{R}$. Torsion: $2 \times 300 \mathrm{NM}$. Rotational velocity: $120 \% \mathrm{~min}$. Flowrate : $2 \times 1 \mathrm{~L} / \mathrm{min}$.

Parameters of the hydraulic cylinder: $\square 125 \times$ $55 \times 140, \mathrm{P}=12 \mathrm{MP}$,

Fup $=2 \times 12 \mathrm{~T}$, Vup $=140 \mathrm{MM} / 120 \mathrm{~S}$ 。Q $=2 \times 2 \mathrm{~L} / \mathrm{MIN}$ 。

Electronic motor: upright marine engine Y100L1-4(2.2Kw $\times 1400 \mathrm{r} / \mathrm{min})$

C. principle and analyses of the main loops

According to the 7 kinds of movements which the boarding ladder should realize, the hydraulic scheme is designed.

1). Hydraulic Cylinder Control Part.

Working Process:

Start the electronic motor, the hydraulic pump will supply hydraulic liquid to the system.

Valve 3 (electromagnetic four way change valve [7], showed in Figure.6) is used to control the ladder's up and down. It is worthwhile to note that if the median position of valve 3 is conducted, the ladder could bob up and down as the waves pass by. The usage of the design is to protect pump and loops in case that the pressure of the system was too high causing damage to hydraulic system.

In case of power off, the hand-operated valve (valve 5 and valve 6) is installed in the hydraulic system. If the system turn to manual, valve 8 (four way change valve) is to control the ladder's up and down $\&$ bobbing on the waves.

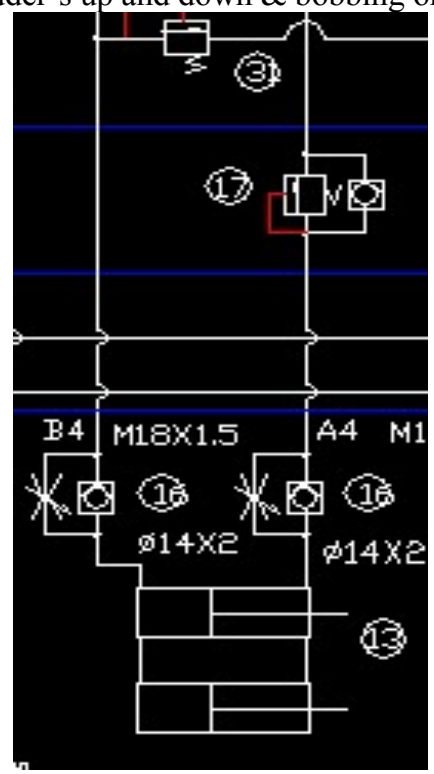

Figure 7. Hydraulic cylinder control part

As shows in Figure.7, the cylinder loop is parallel. Valve 16 (unidirectional throttle valve ) is used to control the velocity of the ladder's titling up and down. Valve 17 (reiterative one-way sequence valve) is used to control the 
pressure of the system so that the ladder could stop at any angle in the range of $\pm 30^{\circ}$.

2). Gyration Control Part.

Valve 1 (four way change valve) controls the ladder's rotation. It should be noted that if the median position is conducted, the ladder would be properly positioned. In addition, the function of hand-operate valve 2 (four way change valve) is to control the ladder's bobbing up and down on waves.

Valve 1 is also a proportional valve. The moment of inertia of the ladder is very large, so valve 1 is to guarantee the smoothness of rotation process of the ladder. What's more valve 1 can also regulate the velocity of rotation.

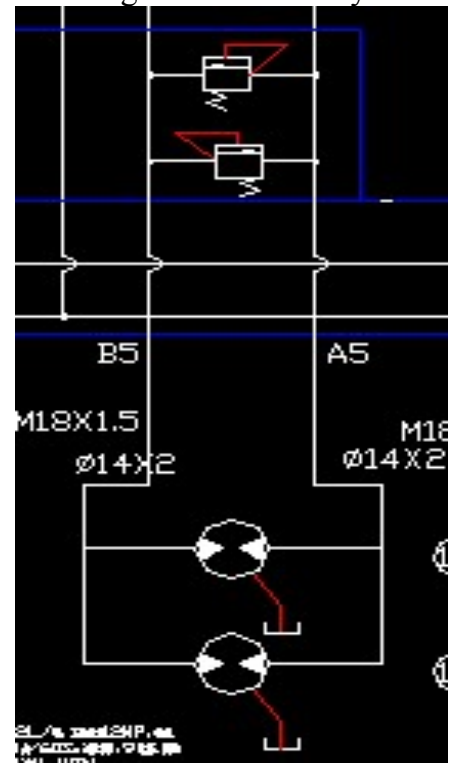

Figure 8. Hydraulic motor control part

High-pressure safety valve (showed in Figure.8) is installed in the hydraulic motor loop. It is used to make the ladder and the ship to be flexible in case that the ladder crashed into a heavy object such as pier etc., causing high pressure in the hydraulic system and even damage to the ladder. This design will increase the safety of the ladder.

\section{SUMMARIES}

Based on the analysis and optimization of the simple boarding ladder, this paper researches and develops a new hydraulic boarding ladder. It presents the working principle, the compact $\&$ stable structure and 3D-model by CATIA of the new ladder. Compared with the simple boarding ladder, the new boarding is more reliable and safe. Equipped with these advantages, the function of new boarding ladder is much more powerful. Therefore, the application scope is much more extensive.

\section{REFERENCES}

[1] Meifang Xu, 230000 VLOC Out Fitting Design, Journal of Ship Design, 2009.6(1)14 18 .

[2] Tao Liu, Gaorun Qian, Design and Application of a Hydraulic Floating Flat. Hydraulic \& Pneumatic, 2005(2), 45 46.

[3] Gongping Wang, Kong Qing. An analysis on the safety problems of Boarding Machine in the Oil Terminal. Port Techniques,2006(6),36 37

[4] Tao Liu, Dafang Wang, Zhiquan Cui, Yinghu Shao. Design and Application of Passenger Stairs Floating Flat for the Harbor, Machine Tool \&Hydraulics, 2006 (1),102 145.

[5] Chunsheng Li, Yi Zhao, Shiyi Liu, Anhua Zuo. Port Loading and Unloading. 2003 (3),28 29

[6] Difen, Xion, Storace Transportation \& Preservation Commodities.2008 (8),117 118

[7] Hydraulic Power Transmission and Pneumatic Power Transmission, Tsinghua University press, 2005.9.70 100.

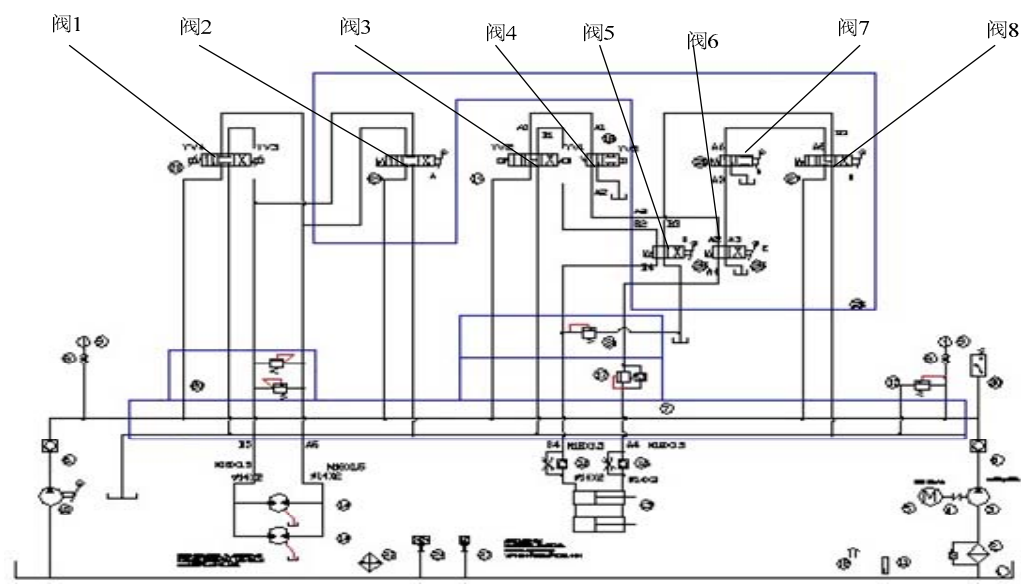

Figure 6. Principle of the hydraulic system 